\title{
Quantum-information distribution via entanglement
}

\author{
Mio Murao, ${ }^{1,2}$ Martin B. Plenio, ${ }^{2}$ and Vlatko Vedral ${ }^{3}$ \\ ${ }^{1}$ Semiconductor Laboratory, The Institute of Physical and Chemical Research (RIKEN), Wako-shi 351-01, Japan \\ ${ }^{2}$ Optics Section, Blackett Laboratory, Imperial College, London SW7 2BZ, United Kingdom \\ ${ }^{3}$ Centre for Quantum Computing, Clarendon Laboratory, University of Oxford, Oxford OX1 3PU, United Kingdom
}

(Received 9 September 1999; published 16 February 2000)

\begin{abstract}
We present a generalization of quantum teleportation that distributes quantum information from a sender's $d$-level particle to $N_{o}$ particles held by remote receivers via an initially shared multiparticle entangled state. This entangled state functions as a multiparty quantum information distribution channel between the sender and the receivers. The structure of the distribution channel determines how quantum information is processed. Our generalized teleportation scheme allows multiple receivers at arbitrary locations, and can be used for applications such as optimal quantum information broadcasting, asymmetric telecloning, and quantum error correction.
\end{abstract}

PACS number(s): 03.67.Hk

\section{INTRODUCTION}

In the quantum teleportation scheme [1], quantum information of an unknown state of a $d$-level particle (an "input" particle) is faithfully transmitted from a sender (Alice) to a remote receiver (Bob) via an initially shared pair of maximally entangled particles. The distributed entangled particles shared by Alice and Bob function as a quantum information channel for the faithful transmission. Quantum teleportation has been demonstrated in several successful experiments [2]. It represents the basic building block of future quantum communication networks between distant parties [3].

In addition to the "one-to-one" quantum communication of teleportation, it is natural to consider "one-to-many" quantum communication via quantum channels, i.e., quantum broadcasting from a sender to several spatially separated receivers. However, it is not possible to perform one-tomany quantum communication perfectly, because the nocloning theorem [4] forbids perfect duplication of quantum information. Approximate methods for quantum cloning are known but these methods require all parties (the original and all the approximate copies) to be in one place.

Our strategy for one-to-many quantum communication is to distribute quantum information of a particle from a sender to many distant receivers. Such a strategy, dubbed quantum telecloning, has been suggested in Ref. [5]. In the quantum telecloning scheme, information of an input qubit (a $d=$ two-level particle) is distributed into $M$ particles which are optimal clones and $M-1$ which are ancilla particles, all spatially separated from each other. This transmission is achieved by first establishing a particular initial entangled state between the sender and receivers. The protocol of quantum telecloning is then similar to that for original quantum teleportation [1], consisting of a joint measurement by Alice, two-bit classical communication from Alice to Bob and a local operation by Bob. This "optimal broadcasting" of quantum information relies on the structure of the distributed entanglement which functions as a one-to-many quantum communication channel. Recently, the telecloning protocol has been generalized to the case of $N(\leqslant M)$ identical input qubits being distributed to $M$ spatially separated parties by
Dür [6]. In this generalization, the same entangled state of Ref. [5] is used for the quantum channel, but a more generalized positive operator valued measure (POVM) is performed for the joint measurement.

We also consider variation of this distribution method for alternative applications. In this paper, we present optimal quantum information broadcasting for $d$-level particles, asymmetric telecloning of qubits, and quantum error correction via entanglement as examples of a generalization of quantum teleportation to one-to-many quantum communication. The important rule of our game is that the receivers are spatially separated from each other so that we do not allow any global operations among receivers.

There is an alternative trivial way to distribute quantum information from a sender to many receivers if we allow the sender to run quantum networks that involve global operations of many particles. In this case, the sender first performs quantum networks for encoding one particle information into several particles in her site. Then she transmits the encoded particles to each receiver using the original teleportation scheme with two particle maximally entangled state [1]. For the transmission, $M \log _{2} d$ ebits of entanglement are required to distribute quantum information of a $d$-level particle into the spatially separated $M$ receivers. The sender performs the measurement $M$ times and uses $M d^{2} / 2$ bits of classical communication from the sender to receivers. On the other hand, in our direct information distribution scheme, multiparticle entanglement is used simultaneously for both encoding information and transmission. We need only a single joint measurement and require only $d^{2} / 2$ bits of classical communication (announced publically to all the receivers). The amount of entanglement between the sender and the receiver as the whole is $\log _{2} d$ ebits. Thus our direct information distribution via entanglement is more efficient in terms of local and global operations, classical communication, and the resource of entanglement.

Distributing information to several different parties can be useful for protecting against eavesdropping. Thus the information distribution can be used for more secure quantum communication. The "tele-error-correction" scheme will provide us with another interpretation of quantum error cor- 
rection and an interesting observation about entanglement required for the quantum channel for encoding. Our information distribution scheme can be also functions as a "ready made network" [7] when all the particles of the quantum channel are at one site, say Alice's site. The quantum channel is then a "black box" having an input port and several output ports to encode a single particle state into a multiparticle state. The "manufacturer" performs the complicated quantum operations to produce the black box. Alice, the "user," only needs to perform the joint measurement for inputting information, and single particle operations depending on the measurement outcome, instead of several controlled-NOT (CNOT) operations required for the global operations of particles.

The rest of the paper is organized as follows. In Sec. II we present our generalization of quantum teleportation for information distribution. Applications of the information distribution scheme are then presented in the following sections. The optimal quantum information broadcasting $(1 \rightarrow M$ telecloning) for $d$-level particles is shown in Sec. III the asymmetric telecloning for distributing information with different fidelity for each receiver are investigated in Sec. IV. Tele-errorcorrection, quantum error correction via the information distribution scheme, is presented in Sec. V. A summary is given in Sec. VI.

\section{A GENERALIZATION OF QUANTUM TELEPORTATION}

In the quantum teleportation scheme of Bennett et al. [1], a pair of maximally entangled particles ( $d$-level particles) initially shared by a sender (Alice) and a receiver (Bob) functions as a channel for quantum information with the help of a classical information channel. Alice's particle is used as a "port" for information input, and Bob's particle is used as the "output" port in the scheme. We may imagine that there are two processes taking place during the faithful transmission of quantum information of an unknown state of an "input particle" from Alice to Bob. The first process is the "information input" process. Alice performs a joint measurement in the maximally entangled basis of the input particle and her port particle. Alice obtains one of the $d^{2}$ possible measurement results. This operation "injects" quantum information from the input particle into the quantum channel. We call this measurement a "Bell-type measurement" not only in the context of qubits $(d=2)$, but in general for $d$-level particles and the maximally entangled basis is called the Bell basis. Injected information appears at Bob's output particle as one of the $d^{2}$ orthogonal states depending on the result of the Bell-type measurement. Without information about Alice's measurement result, the output particle of Bob is in an equal mixture of $d^{2}$ orthogonal states, which does not provide any information about the original states. (If the output state of Bob gave any information of Alice's input before receiving the measurement result from Alice, Alice and Bob could communicate faster than light!) Thus we need a second process, the "recovery unitary operation" (RUO). In this process, Alice notifies which of the $d^{2}$ possible measurement results she obtained. Then Bob performs a unitary operation on the output particle depending on the measurement result to recover the quantum information of the input particle.

Now we generalize the quantum teleportation scheme for distributing quantum information of a $d$-level particle to more than one receiver via a multiparty quantum channel. The quantum channel is a multiparticle entangled state initially shared between the sender and the receivers (Bob, Charlie, and so on). The sender and receivers are spatially separated from each other and no global operation between particles held by different receivers is allowed.

In our scheme, the input, port and output particles of the original teleportation scheme are replaced by groups of input particles, port particles, and output particles. We represent the number of the input, port and output particles as $N_{i}, N_{p}$, and $N_{o}$, respectively. To implement quantum information of one $d$-level particle in the groups of particles, we "use" a $d$-dimensional subspace for each group of $d$-level particles. We denote the basis of the $d$-dimensional subspace as $\left\{\left|\psi_{j}\right\rangle\right\}$ for the input particles, $\left\{\left|\pi_{j}\right\rangle\right\}$ for the port particles, and $\left\{\left|\phi_{j}\right\rangle\right\}$ for the output particles, where $j=0,1, \ldots, d-1$. All these bases are represented by the states of (multi)particles. For example, information of a $d$-level particle implemented in the sender's $N_{i}$ input particles is represented as

$$
|\psi\rangle=\sum_{j=0}^{d-1} a_{j}\left|\psi_{j}\right\rangle
$$

under the constraint $\Sigma\left|a_{j}\right|^{2}=1$.

The quantum channel for information distribution between the sender and the receivers is a maximally entangled state of the sender's port particles and the receivers' output particles:

$$
|\xi\rangle=\frac{1}{\sqrt{d}} \sum_{j}\left|\pi_{j}\right\rangle \otimes\left|\phi_{j}\right\rangle .
$$

The joint state of the input particle and the channel is

$$
\begin{aligned}
|\psi\rangle \otimes|\xi\rangle= & \sum_{n} \sum_{m}\left|\Phi_{n m}\right\rangle \\
& \otimes \frac{1}{\sqrt{d}} \sum_{j}^{d-1} \exp [-2 \pi i j n / d] \alpha_{j}\left|\phi_{\overline{j+m}}\right\rangle,
\end{aligned}
$$

where $\overline{j+m}=[(j+m) \bmod d]$ and $\left|\Phi_{n m}\right\rangle$ is a joint state of the input particles and the port particle in a maximally entangled basis (the Bell-type basis)

$$
\left|\Phi_{n m}\right\rangle=\frac{1}{\sqrt{d}} \sum_{k}^{d-1} \exp [2 \pi i k n / d]\left|\psi_{k}\right\rangle \otimes\left|\pi_{k+m}\right\rangle
$$

for $0 \leqslant n, m \leqslant d-1$. Therefore the RUO for a Bell-type measurement outcome $\left|\Phi_{n m}\right\rangle$ is given by

$$
U_{n m}=\sum_{j} \exp [2 \pi i j n / d]\left|\phi_{j}\right\rangle\left\langle\phi_{\overline{j+m}}\right|
$$


So far we have just relabeled the basis of the input and output particles from the single particle computational basis $|j\rangle$ into the multiparticle basis $\left|\psi_{j}\right\rangle,\left|\pi_{j}\right\rangle$, and $\left|\phi_{j}\right\rangle$. It is remarkable in our generalization for quantum information distribution that the RUO $U_{n m}$ can be replaced by a local recovery unitary operation (LRUO) $U_{n m}^{\text {local }}$, which is a direct product of local operations for each particle:

$$
U_{n m}^{\text {local }}=\mathcal{U}_{n m}^{1} \otimes \cdots \otimes \mathcal{U}_{n m}^{N_{o}},
$$

where $\mathcal{U}_{n m}^{l}$ denotes the local operation for the $l$ th particle of the receivers, under certain conditions for the output state basis $\left\{\left|\phi_{i}\right\rangle\right\}$ which we will describe later in this section. Although $U_{n m}^{\text {local }} \neq U_{n m}$ in general for the full Hilbert space for the $N_{o}$ particles, $U_{n m}^{\text {local }}$ operates in the same way as the RUO $U_{n m}$ in Eq. (5) on the subspace spanned by the output state basis $\left\{\left|\phi_{j}\right\rangle\right\}$,

$$
U_{n m}^{\text {local }}\left|\phi_{j}\right\rangle=U_{n m}\left|\phi_{j}\right\rangle,
$$

for any $j$. We note that the LRUO Eq. (6) is not always determined uniquely for the corresponding (global) RUO defined by Eq. (5). The condition that the RUO is local places additional constraints on the output state basis.

Since the RUO $U_{n m}$ can be decomposed into the products of $U_{01}$ and $U_{10}$ from the definition of Eq. (5), the LRUO $U_{n m}^{\text {local }}$ may be decomposed in the similar manner:

$$
U_{n m}^{\text {local }}=\underbrace{U_{10}^{\text {local }} \ldots \cdot U_{10}^{\text {local }}}_{n \text { times }} \underbrace{U_{01}^{\text {local }} \cdot \ldots \cdot U_{01}^{\text {local }}}_{m \text { times }} .
$$

Then the condition for the output state basis is the existence of the following two LRUO's:

$$
U_{01}^{\text {local }}\left|\phi_{j}\right\rangle=\mathcal{U}_{01}^{1} \otimes \cdots \otimes \mathcal{U}_{01}^{N_{o}}\left|\phi_{j}\right\rangle=\left|\phi_{j-1}\right\rangle,
$$

which changes the state from $\left|\phi_{j}\right\rangle$ to $\left|\phi_{\overline{j-1}}\right\rangle$, and

$$
U_{10}^{\text {local }}\left|\phi_{j}\right\rangle=\mathcal{U}_{10}^{1} \otimes \cdots \otimes \mathcal{U}_{10}^{N_{o}}\left|\phi_{j}\right\rangle=\exp [2 \pi i j n / d]\left|\phi_{j}\right\rangle,
$$

which changes the phase depending on the state $\left|\phi_{j}\right\rangle$.

The protocol for distributing quantum information from a sender to spatially separated receivers,

$$
|\psi\rangle_{\text {sender }}=\sum_{j=0}^{d-1} a_{j}\left|\psi_{j}\right\rangle \rightarrow|\phi\rangle_{\text {receivers }}=\sum_{j=0}^{d-1} \alpha_{j}\left|\phi_{j}\right\rangle,
$$

via the quantum channel defined by Eq. (2) is the following:

1. The sender performs a Bell-type measurement on the input particles and the port particles in the basis $\left\{\left|\Phi_{n m}\right\rangle\right\}$. We expect $d^{2}$ different measurement outcomes.

2. The sender classically (and publicly) broadcasts the measurement outcome (on which basis of $\left|\Phi_{n m}\right\rangle$ she obtained by the projection) to the receivers.

3 . Depending on the broadcast result $\left|\Phi_{n m}\right\rangle$, the receivers perform the LRUO $U_{n m}^{\text {local }}$.
Information of an initial state $|\psi\rangle$ (of $d$-level system) is faithfully transmitted via the quantum channel to an encoded state $|\phi\rangle$ which is the state of the particles distributed among $N_{o}$ spatially separated receivers. This "teleportation" of quantum information of a $d$-level particle is faithful because the channel represented by Eq. (2) has $\log _{2} d$ ebit entanglement between the sender and the receivers as the whole. The appearance of the output information at each receiver's particle is the result of the information distribution. Distribution of information depends on properties of the output state basis $\left|\phi_{j}\right\rangle$.

This generalization of quantum teleportation looks simple in this representation. However, as we will show later, it has more applications. Optimal quantum information broadcasting and asymmetric telecloning are just special cases of the scheme. Also quantum error correction can be carried out via entanglement with additional conditions on the output state and a slight extension of the concept of the Bell-type measurement in the decoding process.

\section{OPTIMAL BROADCASTING FOR MULTILEVEL PARTICLES}

Although information in an unknown quantum state cannot be copied perfectly (no-cloning theorem) [4], a way has been found to obtain "optimal" copies of the original state by an global unitary transformation involving several particles [8]. The optimality of copies is defined by ensuring the largest fidelity from the original state. This quantum optimal cloning of qubits ( $d=2$ particles) has been studied in Refs. [8-11]. While optimal cloning transformations involve global operations on qubits, we have recently considered the problem of quantum "telecloning" for qubits $(d=2)$ in Ref. [5]. Telecloning is a combination of the universal optimal cloning and quantum teleportation performed simultaneously. The aim of telecloning is to broadcast information of an unknown state from a sender to several spatially separated receivers exploiting an entangled state as a quantum channel. The properties of the quantum channel for the qubit telecloning has been investigated in Ref. [5].

For the more general case, the problem of optimal cloning of $N$ identical unknown input states to $M$ output copies of $d$-level particles, which is called " $N \rightarrow M$ optimal quantum cloning" is formulated in Ref. [12]. In that paper, Werner has shown that the optimal cloning map $\hat{T}$ to obtain $M$ optimal clones from $N$ identical (unknown) input states is the projection of the direct product of the $N$ input states and $M$ $-N$ identity states onto the symmetric subspace of $M$ particles:

$$
\hat{T}(\rho)=\frac{d[N]}{d[M]} s_{M}\left(\rho \otimes 1^{\otimes(M-N)}\right) s_{M}
$$

where $s_{M}$ is the projection operator for the symmetrized state of $M d$-level particles, $\rho$ is the density operator for the input state given by the direct product of an input state $|\psi\rangle\langle\psi|$, 


$$
\rho=\underbrace{|\psi\rangle\langle\psi|\otimes \cdots \otimes| \psi\rangle\langle\psi|}_{n \text { times }},
$$

and $d[N]$ is the number of the symmetrized state for $N$ $d$-level particles given by $d[N]={ }_{d+N+1} \mathrm{C}_{N}$. Werner has proved the optimality of the clones obtained by the cloning map Eq. (12) mathematically. The cloning map is the completely positive, trace preserving map. However, physical implementation of the optimal cloning map is not obvious, since the cloning map (12) is not a unitary transformation. To find the corresponding unitary transformation for the 1 $\rightarrow M$ optimal cloning of $d$-level particles, we need a pure state representation including ancilla particles. (For a special case of $M=2$, the unitary transformation of the optimal cloning map for $d$-level particles has been obtained by Bužek and Hillery [9].)

In this section, we present optimal quantum information broadcasting for multilevel particles $(1 \rightarrow M$ telecloning of a $d$-level particle), as an application of the quantum information distribution scheme described in the previous section. In our scheme, we obtain the pure state representing $M$ optimal clones of an unknown state and $M-1$ ancillas. Consequently, we find the unitary transformation which implements the Werner's optimal cloning map for $d$-level particles [12].

For optimal quantum information broadcasting, the input state basis and the port state basis are taken in a single particle computational basis $\{|j\rangle\}$. The output state basis is represented by the states $\left\{\left|\phi_{j}\right\rangle\right\}(j=0,1, \ldots, d-1)$ consisting of $N_{o}=2 M-1 d$-level particles where $M-1$ particles are ancillas and $M$ particles are for presenting optimal cloning states at the end of the protocol.

The output state basis consisting of $2 M-1$ particles is represented in terms of the normalized and the symmetrized state $\left|\xi_{k}^{M}\right\rangle$ of $M d$-level particles,

$$
\left|\phi_{j}\right\rangle=\frac{\sqrt{d}}{\sqrt{d[M]}} \sum_{k=0}^{d[M]-1}{ }_{P}\left\langle j \mid \xi_{k}^{M}\right\rangle_{P A} \otimes\left|\xi_{k}^{M}\right\rangle_{C},
$$

where $P$ denotes the port particles, $A$ denotes the $M-1$ ancilla particles, and $C$ denotes the $M$ particles for optimal clones. The structure of the symmetrized state is the key feature for our optimal quantum information broadcasting as we will show later. In the computational basis, the symmetrized state $\left|\xi_{k}^{M}\right\rangle$ can be represented by

$$
\left|\xi_{k}^{M}\right\rangle=\frac{1}{\sqrt{\mathcal{N}\left(\xi_{k}^{M}\right)}}\left|\mathcal{P}\left(a_{0}, a_{1}, \ldots, a_{M-1}\right)\right\rangle,
$$

where $\mathcal{P}$ denotes an operator which creates the sum of all possible states represented by permutation of the elements $\left\{a_{0}, \ldots, a_{M-1}\right\}$ for $a_{n} \in\{0,1, \ldots d-1\}$ and $a_{n+1} \geqslant a_{n}$ under the constraint $\mathcal{N}\left(\xi_{k}^{M}\right)$ imposing the normalization of $\left|\xi_{k}^{M}\right\rangle$. The index $k$ for the symmetrized state is defined by the following: First we assign to each string $\left\{a_{0}, \ldots, a_{M-1}\right\}$ a number

$$
h\left(a_{0}, a_{1}, \ldots, a_{M-1}\right)=\sum_{n=0}^{M-1} a_{n} d^{M-1-n} .
$$

Then we sort those numbers in increasing order. The index $k$ $(0 \leqslant k \leqslant d[M])$ is then associated with the string $\left\{a_{0}, \ldots, a_{M-1}\right\}$

$$
k=f_{M}\left(a_{0}, \ldots, a_{M-1}\right)
$$

giving rise to the $(k+1)$ th smallest number $h\left(a_{0}, a_{1}, \ldots, a_{M-1}\right)$.

The LRUO's for the output state basis $\left\{\left|\phi_{j}\right\rangle\right\}$ are given by

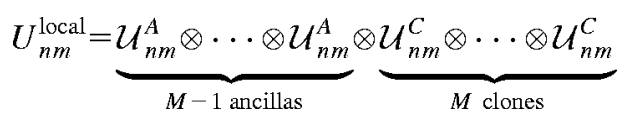

where

$$
\mathcal{U}_{n m}^{A}=\sum_{j=0}^{d-1} \exp [-2 \pi i j n / d]|j\rangle \otimes\langle\overline{j+m}|
$$

and

$$
\mathcal{U}_{n m}^{C}=\sum_{j=0}^{d-1} \exp [2 \pi i j n / d]|j\rangle \otimes\langle\overline{j+m}|
$$

which has the complex conjugate phase of Eq. (20).

The quantum channel is the maximally entangled state between the port particle and the output state particles

$$
|\xi\rangle=\frac{1}{\sqrt{d}} \sum_{j=0}^{d-1}|j\rangle_{P} \otimes\left|\phi_{j}\right\rangle .
$$

It can also be represented in terms of the symmetrized states as

$$
|\xi\rangle=\frac{1}{\sqrt{d[M]}} \sum_{k=0}^{d[M]-1}\left|\xi_{k}^{M}\right\rangle_{P A} \otimes\left|\xi_{k}^{M}\right\rangle_{C} .
$$

The two groups of particles in the information distribution channel, the $P A$ group and the $C$ group, are symmetric to each other. This symmetry property leads to an LRUO $U_{n m}^{\text {local }}$, which is the product of the local operations given by Eqs. (18)-(20). The quantum channel is a maximally entangled state of $(d[M])$-level particles between the $P A$ group and the $C$ group.

As we have shown in the previous paper [5], for the case of $d=2$ (i.e., for qubits), only the $M$ receivers' clone qubits in the quantum broadcasting channel are "directly" entangled to the port qubit according to the Peres-Horodecki criterion [13]. If the partial transpose of the density operator is not positive, the two particles are entangled and otherwise, they are disentangled. The "structure" based on the two particle entanglement of the quantum channel is essential for the optimal quantum information broadcasting of $d$-level particles like in the qubit case. However, because the PeresHorodecki criterion is only valid for the case of limited di- 
mensional particles (two qubits, or entanglement of a qubit and a "qutrit"), the necessity of the two particle entanglement for the distribution of quantum information is still a conjecture.

Exploiting the communication channel given by Eq. (21) and following the protocol for the quantum information distribution described in the previous section, an unknown input state of the sender

$$
|\psi\rangle=\sum_{j=0}^{d-1} \alpha_{j}|j\rangle
$$

is remotely "encoded" into the output state

$$
|\phi\rangle=\sum_{i=0}^{d-1} \alpha_{j}\left|\phi_{j}\right\rangle
$$

held by the $2 M-1$ specially separated receivers via the quantum channel. This output state represents the $M-1$ ancillas and the $M$ optimal clones. As we will show in the following, the reduced density matrix for $M$ optimal clones coincides with a special case $(N=1)$ of the $N \rightarrow M$ universal optimal cloning state for $d$-level particles, which was proved by Werner [12].

The key property of the symmetrized state for our proof is that the symmetric state of $M$ particle can be decomposed into single particle states and symmetric states of the other $M-1$ particles:

$$
\begin{aligned}
\left|\xi_{k}^{M}\right\rangle= & \frac{1}{\sqrt{\mathcal{N}\left(\xi_{k}^{M}\right)}} \sum_{a_{j} \in\{0, \ldots, d-1\}}\left|a_{j}\right\rangle \mid \\
& \left.\times \mathcal{P}_{M-1}\left(a_{0}, \ldots, a_{j-1}, a_{j+1}, \ldots, a_{M-1}\right)\right\rangle \\
= & \frac{1}{\sqrt{\mathcal{N}\left(\xi_{k}^{M}\right)}} \sum_{a_{j} \in\{0, \ldots, d-1\}} \sqrt{\mathcal{N}\left(\xi_{k^{\prime}}^{M-1}\right)}\left|a_{j}\right\rangle\left|\xi_{k^{\prime}}^{M-1}\right\rangle,
\end{aligned}
$$

where $k^{\prime}=f_{M}\left(a_{0}, \ldots, a_{j-1}, a_{j+1}, \ldots, a_{M-1}\right)$. The sum in Eq. (25) is a special sum, it is taken only for different values of $a_{j} \in\{0, \ldots, d-1\}$ (if $a_{j}=a_{j^{\prime}}$, only the smaller index $j$ $<j^{\prime}$ is kept in the sum). To make the relationship between the index $k$ and $k^{\prime}$ clearer, we define another function $g$ that gives the index $k$ of the symmetrized state of $M$ particles when a value of the particle $a_{j}$ is inserted in the $(j-1)$ th position of a symmetrized state of $M-1$ particle having the index $k^{\prime}$ :

$$
k=g\left(a_{j}, k^{\prime}\right) .
$$

Then the output state basis Eq. (14) is represented by

$$
\left|\phi_{i}\right\rangle=\frac{\sqrt{d}}{\sqrt{d[M]}} \sum_{k^{\prime}=0}^{d[M-1]-1} \mathcal{R}_{i}^{k^{\prime}}\left|\xi_{k^{\prime}}^{M-1}\right\rangle_{A} \otimes\left|\xi_{g\left(i, k^{\prime}\right)}^{M}\right\rangle_{C},
$$

where

$$
\mathcal{R}_{i}^{k^{\prime}}=\frac{\sqrt{\mathcal{N}\left(\xi_{k^{\prime}}^{M-1}\right)}}{\sqrt{\mathcal{N}\left(\xi_{g\left(i, k^{\prime}\right)}^{M}\right)}} .
$$

A detailed derivation of the Eq. (27) is found in the Appendix.

The reduced density matrix of the clones is obtained by tracing over the ancilla variables

$$
\begin{aligned}
\rho_{C} & =\operatorname{tr}_{A}|\phi\rangle\langle\phi| \\
& =\sum_{l=0}^{n_{M-1}-1}{ }_{A}\left\langle\xi_{l}^{M-1} \mid \phi\right\rangle\left\langle\phi \mid \xi_{l}^{M-1}\right\rangle_{A} \\
& =\frac{d}{n_{M}} \sum_{i=0}^{d-1} \sum_{i^{\prime}=0}^{d-1} \sum_{k^{\prime}=0}^{d[M]-1} \alpha_{i} \alpha_{i^{\prime}}^{*} \mathcal{R}_{i}^{k^{\prime}} \mathcal{R}_{i^{\prime}}^{k^{\prime}}\left|\xi_{g\left(i, k^{\prime}\right)}^{M}\right\rangle_{C}\left\langle\xi_{g\left(i^{\prime}, k^{\prime}\right)}^{M}\right|
\end{aligned}
$$

The projection operator to the symmetric subspace of $M$ particles in Werner's cloning map given by Eq. (12) in our notation is

$$
s_{M}=\sum_{k=0}^{d[M]}\left|\xi_{k}^{M}\right\rangle\left\langle\xi_{k}^{M}\right|
$$

Then the density matrix for $1 \rightarrow M d$-level optimal clones obtained by Werner [12] is represented as

$$
\begin{aligned}
\rho_{C}= & \hat{\mathrm{T}}(|\psi\rangle\langle\psi|) \\
= & \frac{n_{1}}{d[M]} s_{M}|\psi\rangle\langle\psi| \otimes \mathbf{1}^{\otimes M-1} s_{M} \\
= & \frac{d}{d[M]} \sum_{i=0}^{d-1} \sum_{i^{\prime}=0}^{d-1} \sum_{k^{\prime}=0}^{n_{M-1}-1} \alpha_{i} \alpha_{i^{\prime}}^{*} \mathcal{R}_{i}^{k^{\prime}} \mathcal{R}_{i^{\prime}}^{k^{\prime}} \\
& \times\left|\xi_{g\left(i, k^{\prime}\right)}^{M}\right\rangle_{C}\left\langle\xi_{g\left(i^{\prime}, k^{\prime}\right)}^{M}\right| .
\end{aligned}
$$

This density matrix coincides with our reduced density matrix for the clones Eq. (29). Thus the output state $|\phi\rangle$ given by Eq. (24) represents the optimal cloning state consisting of $M-1$ ancillas and $M$ optimal clones.

\section{ASYMMETRIC TELECLONING}

Quantum telecloning described in the previous section distributes information from an input state evenly to distant receivers. However, it may be desirable to distribute information unevenly to the receivers. For example, if we trust Alice more than Bob, we may decide to distribute more information to Alice. Asymmetric quantum telecloning distributes information from an unknown input particle into several different parties with different fidelity for each party. The corresponding local operation for this information distribution is the asymmetric cloning proposed by Refs. [14] and [15]. In this section, we show an example of $1 \rightarrow 2$ asymmetric telecloning for qubits $(d=2)$.

For asymmetric telecloning, the input state basis is taken as the one qubit computational basis $\{|j\rangle\}$. The output basis 


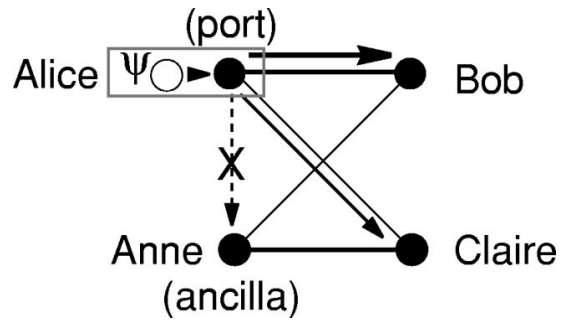

FIG. 1. An asymmetric telecloning state. The width of lines between two particles represents the "strength" of entanglement between the two particles. The difference of strength of entanglement causes asymmetric telecloning.

consist of three qubits, one ancilla qubit $A$ held by Anne (note: she is not Alice) and two clone qubits $B$ and $C$ held by the receivers, Bob and Claire, as

$$
\begin{aligned}
& \left|\phi_{0}\right\rangle=\frac{1}{\sqrt{\mathcal{N}}}(|000\rangle+p|101\rangle+q|110\rangle), \\
& \left|\phi_{1}\right\rangle=\frac{1}{\sqrt{\mathcal{N}}}(|111\rangle+p|010\rangle+q|001\rangle),
\end{aligned}
$$

where $q=1-p, \mathcal{N}$ is a normalization factor given by $\mathcal{N}$ $=1+p^{2}+q^{2}$ and the order of the qubits is $\{A, B, C\}$. The LRUO's are given by $U_{01}^{\text {local }}=\sigma_{z} \otimes \sigma_{z} \otimes \sigma_{z}$ and $U_{10}^{\text {local }}=$ $\sigma_{x} \otimes \sigma_{x} \otimes \sigma_{x}$. The information distribution channel for asymmetric telecloning, which is a maximally entangled state of the port qubit and the output basis $\phi_{0}$ and $\phi_{1}$, is given by

$$
\begin{aligned}
|\xi\rangle= & \frac{1}{\sqrt{2}}\left(|0\rangle\left|\phi_{0}\right\rangle+|1\rangle\left|\phi_{1}\right\rangle\right) \\
= & \frac{1}{\sqrt{2 \mathcal{N}}}\{|0000\rangle+|1111\rangle+p(|0101\rangle+|1010\rangle) \\
& +q(|0110\rangle+|1001\rangle)\} .
\end{aligned}
$$

The channel can be illustrated as follows (Fig. 1) in the case $p>q$.

The information distribution channel for symmetric telecloning is, of course, given by the choice of parameters $p$ $=q=1 / 2$. If we choose $p=0$ or $q=0$, the asymmetric telecloning state consist of two maximally entangled pairs Einstein-Podolsky-Rosen (EPR) pairs]. In this case, the receiver who is sharing the EPR pair with Alice obtains faithful information of the input state and the other, who is sharing the EPR pair with Anne, obtains no information at all. We now investigate how the parameters control the asymmetric distribution of quantum information via entanglement.

Our generalized teleportation protocol with the choice of the distribution channel (33) maps the unknown input state $|\psi\rangle=\alpha_{0}|0\rangle+\alpha_{1}|1\rangle$ to the three qubit state:

$$
|\phi\rangle_{A B C}=\alpha_{0}\left|\phi_{0}\right\rangle+\alpha_{1}\left|\phi_{1}\right\rangle \text {. }
$$

The asymmetric clones are represented by the reduced density matrices

$$
\begin{aligned}
\rho_{B} & =\operatorname{tr}_{A C}|\phi\rangle\langle\phi| \\
& =\frac{1+p^{2}}{\mathcal{N}}|\psi\rangle\left\langle\psi\left|+\frac{q^{2}}{\mathcal{N}}\right| \psi^{\perp}\right\rangle\left\langle\psi^{\perp}\right|
\end{aligned}
$$

for Bob's clone and

$$
\begin{aligned}
\rho_{C} & =\operatorname{tr}_{A B}|\phi\rangle\langle\phi| \\
& =\frac{1+q^{2}}{\mathcal{N}}|\psi\rangle\left\langle\psi\left|+\frac{p^{2}}{\mathcal{N}}\right| \psi^{\perp}\right\rangle\left\langle\psi^{\perp}\right|
\end{aligned}
$$

for Claire's clone, where $\left|\psi^{\perp}\right\rangle$ represents a state orthogonal to the input state $|\psi\rangle$.

To investigate the structure of the quantum channel for asymmetric telecloning based on two-particle entanglement, we calculate the Peres-Horodecki criterion [13]. For asymmetric telecloning, the Peres-Horodecki criterion for the reduced density matrix of the port qubit and Bob's qubit (for the asymmetric clone) $\rho_{P B}$ is

$$
c_{B}(p)=\frac{1-4 p+p^{2}}{4\left(1-p+p^{2}\right)}
$$

and that for the reduced density matrix for the port qubit and Claire's qubit $\rho_{P C}$ is

$$
c_{C}(p)=\frac{-2+2 p+p^{2}}{4\left(1-p+p^{2}\right)} .
$$

There is an interesting case, $c_{C}=0$, which is given for the parameter $p=\sqrt{3}-1$. In this case, the port qubit and the clone qubit of Claire are not directly entangled with each other and the fidelities of the clones, which is the matrix element of the reduced density matrix in terms of the input state $\left\langle\psi\left|\rho_{B}\right| \psi\right\rangle$, are

$$
f_{B}=\frac{2}{3}+\frac{\sqrt{3}}{6}
$$

for Bob's asymmetric clone and

$$
f_{C}=\frac{2}{3}
$$

for Claire's asymmetric clone. The state of fidelity $2 / 3$ is obtained in the classical limit [16]. That is, only the "classical" information of the input state $\psi$ is transmitted via this disentangled channel.

Here we note that some classical information of the input state has also been transmitted to the ancilla qubits of Anne. Since the reduced density matrix of the ancilla qubit is given by

$$
\begin{aligned}
\rho_{A} & =\operatorname{tr}_{B C}|\phi\rangle\langle\phi| \\
& =\frac{1}{\mathcal{N}}|\psi\rangle\left\langle\psi\left|+\frac{p^{2}+q^{2}}{\mathcal{N}}\right| \psi^{\perp}\right\rangle\left\langle\psi^{\perp}\right|,
\end{aligned}
$$


the ancilla qubit (after the telecloning protocol) can be considered to be a "clone" of very low quality, the fidelity $1 / \mathcal{N} \leqslant 2 / 3$, where the equality is taken at $p=q=1 / 2$ (symmetric telecloning). The ancilla qubits only contains classical information of the input state. For the asymmetric case, the ratio of the fidelity of the clones for Bob, Claire, and Anne is $\left[1+p^{2}\right]:\left[1+(1-p)^{2}\right]: 1$, and Anne always keeps a "junk" clone which only contains some classical information of the input state irrespective of the parameter $p$.

As pointed out by Dür [6], the reduced density matrix of the symmetric telecloning state of the port and clone qubits $\rho_{P B}$ is a Werner state $\rho_{W}$ [12]. A Werner state is a state which is diagonal in the maximally entangled state basis $\left\{\Phi^{+}=\Phi_{00}, \quad \Phi^{-}=\Phi_{01}, \quad \Psi^{+}=\Phi_{10}, \quad \Psi^{-}=\Phi_{11}\right\}$. The largest diagonal element of $\rho_{P B}$ (fidelity) is

$$
\left\langle\Phi^{+}\left|\rho_{W}\right| \Phi^{+}\right\rangle=\frac{3(M+1)}{6 M} .
$$

Thus if we only "see" the port qubit and the one of the receivers' qubit, $1 \rightarrow M$ (symmetric) quantum telecloning is equivalent to the standard teleportation using an imperfect quantum channel made of the Werner state $\rho_{W}$. For the case of $M=2$, the fidelity of the Werner state is $3 / 4$.

For asymmetric telecloning, the reduced density matrix of the quantum channel is also represented by the Werner state as

$$
\begin{aligned}
\rho_{P B}= & \frac{1}{2 \mathcal{N}^{\prime}}\left\{(1+p)^{2}\left|\Phi^{+}\right\rangle\left\langle\Phi^{+}\right|+q^{2}\left(\left|\Phi^{-}\right\rangle\left\langle\Phi^{-}\right|\right.\right. \\
& \left.\left.+\left|\Psi^{+}\right\rangle\left\langle\Psi^{+}|+| \Psi^{-}\right\rangle\left\langle\Psi^{-}\right|\right)\right\} .
\end{aligned}
$$

This representation of the quantum channel shows the relation between the asymmetric telecloning and Cerf's Pauli cloning machines [14]. Cerf has suggested that a Pauli cloning machine performs as a universal (i.e., independent of input states) asymmetric cloning machine only in the case of depolarizing channels represented by the Werner state.

\section{TELE-ERROR-CORRECTION}

Since decoherence is the main obstacle to quantum information processing, the discovery of quantum error correction schemes [17] is very important for the practical realization of quantum computation and quantum communication. In this section, we show how quantum error correction can be performed via distributed entanglement as another example of our information distribution scheme.

The standard quantum error correction schemes [17] consist of the following four processes.

(i) The first process involves encoding information. Information of a qubit is encoded into a state of $N_{e}$ qubits $\left(N_{e}\right.$ $=3$ for the case that only one kind of error happening to one of the qubits, $N_{e}=5$ or $N_{e}=7$ for the case that one of the three kinds of errors happening to one of the qubits) by an global unitary transformation of $M$ qubits.

(ii) After encoding, you may have an error in one of the encoded qubits. The second process is for decoding information of the state after an error occurs. (iii) The decoding process is performed by a reverse global unitary transformation of encoding. After the decoding process, one of the qubits is an "output qubit" and the others are ancilla qubits which indicates whether an error occurred. The relationship of the states of the ancilla qubits and in which qubit the error occurred is given in the syndrome table.

(iv) The fourth process is to correct errors. We measure the ancilla qubits and correct an error indicated by the measurement result and the syndrome table. Alternatively, some global transformation among the decoded qubits may be performed for error correction instead of measuring ancilla qubits.

The first process, encoding qubit information into a state of many qubits for error correction, is carried out via our information distribution scheme with the appropriate choice of output state basis consisting of $N_{e}$ qubits. We limit ourselves to the case of correction of a single error. Three kinds of errors may happen to a qubit in the encoded state. These are equivalent to the single qubit operation $\sigma_{z}$ (type 1), $\sigma_{x}$ (type 2) or $\sigma_{z} \cdot \sigma_{x}$ (type 3). An error of the type $l$ ( $l$ $=1,2,3)$ happening to the $\eta$ th particle $\left(\eta=1, \ldots N_{e}\right), \epsilon_{l}^{\eta}$, maps from an output state basis $\left|\phi_{j}\right\rangle$ to a state $\left|\varphi_{j}^{\zeta}\right\rangle$. The index $\zeta$ is determined by $\zeta=(l-1) N_{e}+\eta$. We define that $\zeta=0$ represents no error. In some other cases, only one type of errors is expected. In this case, we only need to consider $l=1$. If we denote the total possible types of errors as $L, \zeta$ takes $\left(L N_{e}+1\right)$ different values, $\zeta=0, \ldots, L N_{e}$. The state changes through the encoding process and error as follows:

$$
|\psi\rangle=\sum_{j=0}^{1} \alpha_{j}|j\rangle \rightarrow|\phi\rangle=\sum_{j=0}^{1} \alpha_{j}\left|\phi_{j}\right\rangle \rightarrow|\varphi\rangle=\sum_{j=0}^{1} \alpha_{j}\left|\varphi_{j}^{\zeta}\right\rangle .
$$

For successful information distribution scheme via teleportation, the output state basis is required to have the LRUO $U_{n m}^{\text {local }}$. In addition, it has to satisfy the following condition:

$$
\left\langle\phi_{j^{\prime}}\left|\epsilon_{l^{\prime}}^{\eta^{\prime} \dagger} \epsilon_{l}^{\eta}\right| \phi_{j}\right\rangle=\left\langle\varphi_{j^{\prime}}^{\zeta^{\prime}} \mid \varphi_{j}^{\zeta}\right\rangle=\delta_{j, j^{\prime}} \delta_{\zeta, \zeta^{\prime}}
$$

for error correction (the necessary and sufficient condition for quantum error correction [18]). This condition states that different errors map a state into different states so that it is possible to distinguish different errors. The state after an error indicated by $\zeta$ is not in the subspace of the original output state basis $\left\{\left|\phi_{j}\right\rangle\right\}$ but in the subspace of the $\left\{\left|\varphi_{j}^{\zeta}\right\rangle\right\}$. We now treat the Hilbert space of dimensions $L N_{e}+1$, which is the sum of all subspaces for given $\zeta$, instead of two dimensions for a qubit and the subspace of the output state.

The decoding and the error correction steps can be described by the information distribution scheme analogous to telecloning instead of performing a global unitary operation. We use a pair of maximally entangled qubits for the quantum channel $\left(\left|\xi_{d}\right\rangle=(|00\rangle+|11\rangle) / \sqrt{2}\right)$, and an "extended" Belltype measurement for the enlarged space occupied by the state after an error occurs. The joint state of the error state and the channel $\left|\xi_{d}\right\rangle$ is 


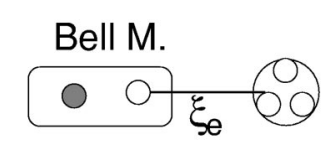

Bell M.

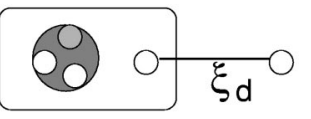

encoding

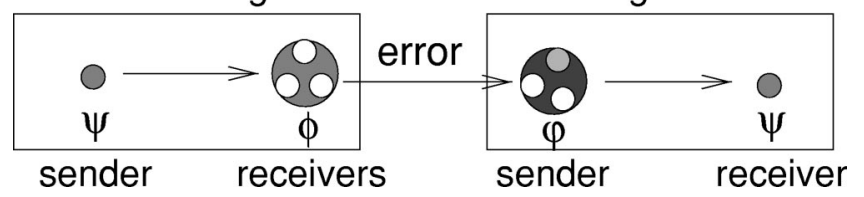

FIG. 2. Amplitude (or phase) error correction via generalized teleportation is illustrated. $\xi_{e}$ denotes the quantum channel for encoding and $\xi_{d}$ denotes the quantum channel for decoding and error correction. The first raw represents the protocol and the second raw represents how quantum information is encoded in each process.

$$
|\varphi\rangle \otimes\left|\xi_{d}\right\rangle=\sum_{n} \sum_{m}\left|\Phi_{n m}^{\zeta}\right\rangle \otimes \frac{1}{\sqrt{2}} \sum_{j}^{1} \exp [-\pi i j n] \alpha_{j}|\overline{j+m}\rangle,
$$

where $\left|\Phi_{n m}^{\zeta}\right\rangle$ denotes the measurement outcomes of the extended Bell type measurement performed by the sender

$$
\left|\Phi_{n m}^{\zeta}\right\rangle=\frac{1}{\sqrt{2}} \sum_{k=0}^{1} \exp [\pi i k n]\left|\varphi_{k}^{\zeta}\right\rangle \otimes|\overline{k+m}\rangle
$$

for $n, m=0,1$ and $\zeta=0, L N_{e}$. There are $4\left(L N_{e}+1\right)$ different outcomes possible measured by the extended Bell measurement. However, we only need information of $n$ and $m$ for finding the appropriate RUO. So the sender only needs to broadcast two bits of classical information to the receiver. The RUO for the output qubit

$$
U_{n m}=\sum_{j=0}^{1} \exp [\pi i j n]|j\rangle\langle\overline{j+m}| .
$$

will give the error corrected original state $|\psi\rangle$ (in a remote place from the error state).

\section{A. Three-qubit code}

To illustrate our tele-error-correction scheme, we present a simple example, a three-qubit error correction code (Fig. 2 ). This code is able to correct an error, which is known to be one of $\left\{\sigma_{z}, \sigma_{x}, \sigma_{z} \cdot \sigma_{x}\right\}$ that happens to one of the qubits in the encoded state. In the following, we investigate the case of an amplitude error (type 2). We start from the encoding process. The output state basis for encoding is the three-qubit state:

$$
\begin{aligned}
& \left|\phi_{0}\right\rangle=|000\rangle, \\
& \left|\phi_{1}\right\rangle=|111\rangle .
\end{aligned}
$$

The LRUO is given by $U_{01}^{\text {local }}=\sigma_{x} \otimes \sigma_{x} \otimes \sigma_{x}$ and $U_{10}^{\text {local }}=\sigma_{z}$ $\otimes \sigma_{z} \otimes \sigma_{z}$ in the computational basis.
The communication channel for encoding is given by the four-particle maximally entangled state of GreenbergerHorne-Zeilinger type (GHZ type):

$$
\left|\xi_{e}\right\rangle=\frac{1}{2}(|0000\rangle+|1111\rangle)
$$

The sender and the receivers follow the information distribution protocol. The sender performs the Bell-type measurement of the input and port qubits and broadcasts the measurement result to the receivers. Depending on the four different measurement outcomes $\left|\Phi_{n m}\right\rangle$, the receivers perform the LRUO's. Then information of the input qubit is encoded into the three-qubit state

$$
|\phi\rangle=\alpha_{0}|000\rangle+\alpha_{1}|111\rangle
$$

For decoding and error correction, we require all the encoded qubits (which may have a phase error) to be at the same site of the port qubit. We exploit a maximally entangled state $\left|\xi_{d}\right\rangle=(|00\rangle+|11\rangle) / \sqrt{2}$ as the quantum channel. The RUO's are given by $U_{01}=\sigma_{x}$ and $U_{10}=\sigma_{z}$. We perform the extended Bell-type measurement with the encoded qubits and the port qubit. After an error occurs, the encoded state is mapped to one of the four different states orthogonal to each other depending on the error index $\zeta(=0,1,2,3)$. For each $\zeta$, we have one of four different Bell measurement outcomes, therefore we have one measurement outcome out of 16 possible joint states. These 16 joint states are equivalent to the 16 maximally entangle states for the four-qubit GHZ-type state. We use the "full" Hilbert space of four qubits for error correction.

If no error occurs, the extended Bell-type measurement projects onto one of the only four states $\left|\Phi_{n m}^{0}\right\rangle=\left|\Phi_{n m}\right\rangle$ $(n, m=0,1)$, the same as in the standard teleportation scheme. If a phase error occurs in the $\eta$ th qubit (out of the three qubits), the phase error interchanges the state $|0\rangle \leftrightarrow|1\rangle$ of the $n$th qubit. The extended Bell-type measurement projects into the $\left|\Phi_{n m}^{\zeta}\right\rangle$, which is different from $\left|\Phi^{ \pm}\right\rangle$or $\left|\Psi^{ \pm}\right\rangle$. We perform the appropriate local operation depending on $n$ and $m$ to the output qubit. Then we decode it back to the original input state $|\phi\rangle$.

Now we investigate the quantum channel for error correction. For the GHZ-type maximally entangled state of $N$ particles, there is no direct entanglement between any two qubits. If we trace out any one of the qubits of the GHZ-type state, the rest is in complete mixture of the two orthogonal states consisting of $N-1$ qubits. We have seen that quantum information is transmitted only via an entangled channel in the previous sections. How can we explain flow of quantum information in our error correction scheme via entanglement? In the information encoding process, quantum information of the input state should not be transmitted into any of the qubits. However, the port qubit is maximally entangled with all the three output qubits. From this fact, we may consider that quantum information is transmitted via entanglement among the three qubits and no information is implemented in the local state of each qubit. 


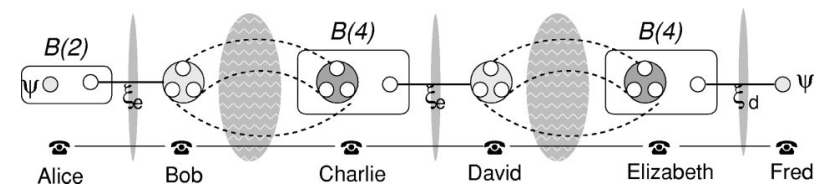

FIG. 3. The error correcting repeater using a combination of the three-qubit error-correction via entanglement. In this figure, $B(2)$ and $B(4)$ represent Bell-type measurement for two and four qubits, respectively. $\xi_{e}$ denotes the quantum channel for encoding (the four-qubit maximally entangled state) and $\xi_{d}$ denotes the quantum channel for decoding (the two qubit maximally entangled state). The dotted lines represent insecure channels. The channels connected through the telephone signs are classical channels.

The tele-error-correction scheme via entanglement channel is a method for quantum communication that is secure against a single-qubit single-type error attack of an eavesdropper. The single-qubit single-type attack of an eavesdropper appears in the encoded state as an error. We can correct the error and retain information of the input state. Thus the attack of the eavesdropper should not gain any information of the input state. We may consider an error correction repeater using a combination of the tele-error-correction schemes (Fig. 3). Here we present an example for an amplitude-type attack, so we do not use the tilded basis. Consider Alice sending quantum information to Fred. Bob, Charlie, David, Elizabeth are located between Alice and Fred and pass through the quantum information. Alice and Bob, Charlie and David, Elizabeth and Fred are separated from each other and connected via secure quantum channels. Bob and Charlie are connected via an insecure channel and so are David and Elizabeth, there may be a single error. Their channels can be nonperfect EPR pairs or even optical fibers with which one may transmit a particle (photon). Alice shares the quantum channel for encoding $\left|\xi_{e}\right\rangle$ with Bob and so does Charlie and David. Elizabeth and Fred share a quantum channel for decoding $\left|\xi_{d}\right\rangle$. Alice performs the Bell-type measurement in the maximally entangled state basis for two qubits denoted by $\mathcal{B}(2)$ and send two-bit classical information to Bob. Bob perform the appropriate (L)RUO of his three qubits. Bob sends information from the encoded three qubits via the insecure channel. Charlie receives the three qubits from Bob. An error might have happen to one of the three qubits. Charlie performs the joint measurement on the three qubits and the port qubit, which he shares with David in the maximally entangled state basis of four qubits denoted by $\mathcal{B}(4)$. Charlie and David follow the protocol of the information distribution scheme. David sends his three qubits via insecure channel to Elizabeth. Finally Elizabeth performs $\mathcal{B}(4)$ together with her three qubits and the port qubit of the quantum channel for decoding. The information of the original state of Alice is now found at the qubit hold by Fred.

We note that the distribution of quantum information for encoding is similar to quantum secret sharing and splitting scheme $[19,20]$ if all the output state qubits are spatially separated as pointed out in Ref. [20]. In Ref. [20], Cleve, Gottesman, and Lo stress that every quantum secret sharing scheme is a quantum error correcting code (in some sense), but that error correction codes are not necessarily quantum secret sharing codes.

\section{B. Correcting amplitude and phase errors}

Here we will show the output state basis for encoding in five-qubit, seven-qubit, nine-qubit quantum error correction codes and their LRUO's. Now the extended Bell-type measurement involves six qubits and eight qubits; it is almost impossible to distinguish all the different outcomes, so it may not be practical, but it is interesting to investigate entanglement of these quantum channels. As we will show in the following, the quantum channel for encoding the fivequbit code requires three-ebit entanglement, the seven-qubit code requires two-ebit entanglement and the nine-qubit code requires one-ebit entanglement. It is interesting that the most condensed error correction code requires the most entanglement.

We first show the seven-qubit quantum error correction code via entanglement because it contains higher symmetry. The two output state basis for encoding are

$$
\begin{aligned}
\left|\phi_{0}\right\rangle= & \frac{1}{2 \sqrt{2}}\{|000\rangle(|0000\rangle+|1111\rangle)+|011\rangle(|0011\rangle \\
& +|1100\rangle)+|101\rangle(|0101\rangle+|1010\rangle) \\
& +|110\rangle(|0110\rangle+|1001\rangle)\}, \\
\left|\phi_{1}\right\rangle= & \frac{1}{2 \sqrt{2}}\{|111\rangle(|0000\rangle+|1111\rangle)+|100\rangle(|0011\rangle \\
& +|1100\rangle)+|010\rangle(|0101\rangle+|1010\rangle) \\
& +|001\rangle(|0110\rangle+|1001\rangle)\} .
\end{aligned}
$$

The LRUO's are given by $U_{10}=\sigma_{z} \otimes \cdots \otimes \sigma_{z}$ and $U_{01}=\sigma_{x}$ $\otimes \cdots \otimes \sigma_{x}$. In this case, one of the even (or odd) order of qubits will be the error corrected using the rest of the qubit.

The broadcasting channel for encoding

$$
\left|\xi_{e}\right\rangle=(1 / \sqrt{2})\left(|0\rangle\left|\phi_{0}\right\rangle+|1\rangle\left|\phi_{1}\right\rangle\right)
$$

can be written in the following two ways:

$$
\begin{aligned}
\left|\xi_{e}\right\rangle= & \frac{1}{4}\{(|0000\rangle+|1111\rangle)(|0000\rangle+|1111\rangle) \\
& +(|0011\rangle+|1100\rangle)(|0011\rangle+|1100\rangle) \\
& +(|0101\rangle+|1010\rangle)(|0101\rangle+|1010\rangle) \\
& +(|0110\rangle+|1001\rangle)(|0110\rangle+|1001\rangle)\} \\
= & (|00\rangle+|11\rangle)(|00\rangle+|11\rangle)(|00\rangle+|11\rangle) \\
& \otimes(|00\rangle+|11\rangle)+(|00\rangle-|11\rangle) \\
& \otimes(|00\rangle-|11\rangle)(|00\rangle-|11\rangle)(|00\rangle-|11\rangle) \\
& +(|01\rangle+|10\rangle)(|01\rangle+|10\rangle)(|01\rangle+|10\rangle)
\end{aligned}
$$




$$
\begin{aligned}
& (|01\rangle+|10\rangle)+(|01\rangle-|10\rangle) \\
& \otimes(|01\rangle-|10\rangle)(|01\rangle-|10\rangle)(|01\rangle-|10\rangle) .
\end{aligned}
$$

Both representations suggest that this state can be considered to be a maximally entangled state of four levels. (The first representation is in two maximally entangled four-level particles and the second is in four maximally entangled fourlevel particles.) Thus the state has $\log _{2} 4=2 e$-bit entanglement.

For the five-qubit error correction code via entanglement, the output state basis for encoding is given by (in the representation of Barenco et al. [21])

$$
\begin{aligned}
\left|\phi_{0}\right\rangle= & |000+111\rangle|00\rangle-|010+101\rangle|11\rangle \\
& +|001+110\rangle|01\rangle+|011+100\rangle|10\rangle, \\
\left|\phi_{1}\right\rangle= & -|000-111\rangle|11\rangle-|010-101\rangle|00\rangle \\
& -|001-110\rangle|10\rangle+|011-100\rangle|01\rangle .
\end{aligned}
$$

In this case, the first qubit will be error corrected using the rest of qubits. The role of qubits are rather asymmetric in this case. The LRUO's are given by

$$
U_{10}=\sigma_{x} \otimes \sigma_{x} \otimes \sigma_{x} \otimes \mathbf{1} \otimes \mathbf{1}
$$

and

$$
U_{01}=-\sigma_{z} \otimes \sigma_{z} \otimes \sigma_{x} \otimes \sigma_{z} \cdot \sigma_{x} \otimes \sigma_{z} \cdot \sigma_{x} .
$$

The quantum channel for this case can be represented by the maximally entangled state of an eight-level system, which contains three ebits of entanglement.

For the nine-qubit error correction code, the encoding output state basis are:

$$
\begin{gathered}
\left|\phi_{0}\right\rangle=|000+111\rangle|000+111\rangle|000+111\rangle, \\
\left|\phi_{1}\right\rangle=|000-111\rangle|000-111\rangle|000-111\rangle .
\end{gathered}
$$

The LRUO's are given by $U_{10}=\sigma_{x} \otimes \cdots \otimes \sigma_{x}$ and $U_{01}=$ $\sigma_{x} \otimes \cdots \otimes \sigma_{x}$ (although these LRUO's are not unique). In this case, any of the qubits in the state can be error corrected using the rest of qubits, so the role of each qubit is very symmetric. The quantum channel for encoding is represented by the maximally entangled state of two-level system, which suggests the amount of entanglement is one ebit.

\section{SUMMARY}

We have presented a generalization of quantum teleportation for distributing quantum information of a $d$-level particle from a sender to $M$ remote receivers via an initially shared multiparticle entangled state. The entangled state functions as a multiparty quantum channel for distributing information. This entangled state is a maximally entangled state between the port particle of the sender and the output particles of the receivers. The existence of two LRUO ( $U_{10}^{\text {local }}$ and $U_{01}^{\text {local }}$ ) for the output state basis is essential for our in- formation distribution to allow multiple receivers at arbitrary locations.

We have presented optimal quantum information broadcasting of a $d$-level particle, asymmetric telecloning of qubits, and tele-error-correction as examples of the quantum information distribution scheme. For the quantum information broadcasting, we show the pure output state for $1 \rightarrow M$ optimal cloning of $d$-level particles including ancillas. This output state is a physical implementation of the optimal cloning map presented by Werner [12]. The investigation of the asymmetric telecloning for qubits suggests that quantum information of the input qubit is only transmitted by a directly entangled channel. The tele-error-correction scheme provides another interpretation of quantum error correction from the viewpoint of entanglement and allows an interesting observation of the amount of entanglement required for the quantum channel for encoding. This scheme can be used for secure communication.

\section{ACKNOWLEDGMENTS}

The authors are grateful to J. Watson for his help during the preparation of this manuscript. We are supported by the Special Postdoctoral Researchers Program of RIKEN, the Leverhulme Trust, and the U.K. Engineering and Physical Sciences Research Council. Parts of this work were supported by the Isaac Newton Institute program "Communication, Complexity and Physics of Information (1999),' and the European Science Foundation-Quantum Information Theory (ESF-QIT) Programme.

\section{APPENDIX}

We show a detailed derivation of Eq. (27). First we show the case $i=0$ and then show the case $i \neq 0$. For the case of $i=0$, the terms which give nonvanishing contribution of the scalar product in Eq. (27) are the terms which contains at least one $\{0\}$ in the computational basis representation. This requires $a_{0}=0$. Only the first $n_{M-1}$ out of $n_{M}$ terms in the symmetrized states $\left|\xi_{k}^{M}\right\rangle$ are $a_{0}=0$ and contribute in the sum of Eq. (27). For $0 \leqslant k \leqslant n_{M-1}-1$, a symmetric state can be decomposed into the two parts:

$$
\begin{aligned}
\left|\xi_{k}^{M}\right\rangle= & \frac{1}{\sqrt{\mathcal{N}\left(\xi_{k}^{M}\right)}}\left|\mathcal{P}_{M}\left(0, a_{1}, \ldots, a_{M-1}\right)\right\rangle_{P A} \\
= & \frac{1}{\sqrt{\mathcal{N}\left(\xi_{k}^{M}\right)}}|0\rangle_{P} \otimes\left|\mathcal{P}_{M-1}\left(a_{1}, \ldots, a_{M-1}\right)\right\rangle \\
& +\frac{1}{\sqrt{\mathcal{N}\left(\xi_{k}^{M}\right)}} \sum_{a_{j} \neq 0}\left|a_{j}\right\rangle \otimes \mid \mathcal{P}_{M-1}\left(0, \ldots, a_{j-1},\right. \\
& \left.\left.a_{j+1}, \ldots, a_{M-1}\right)\right\rangle .
\end{aligned}
$$

The scalar product is now given by 


$$
\begin{aligned}
\sum_{k=0}^{n_{M}-1}{ }_{P}\left\langle 0 \mid \xi_{k}^{M}\right\rangle_{P A} \otimes\left|\xi_{k}^{M}\right\rangle_{C} & \\
= & \sum_{k=0}^{n_{M-1}-1} P\left\langle 0 \mid \xi_{k}^{M}\right\rangle_{P A} \otimes\left|\xi_{k}^{M}\right\rangle_{C} \\
= & \sum_{k=0}^{n_{M-1}-1} \sqrt{\frac{\mathcal{N}\left(\xi_{k}^{M-1}\right)}{\mathcal{N}\left(\xi_{k}^{M}\right)}}\left|\xi_{k^{M-1}}\right\rangle \otimes\left|\xi_{k}^{M}\right\rangle_{C}
\end{aligned}
$$

which is a special case of Eq. (27) with $k=g\left(0, k^{\prime}\right)=k^{\prime}$.

For $i \neq 0$, the decomposition of the $M$ particle symmetrized state in terms of the $M-1$ symmetrized state is

$$
\begin{aligned}
\left|\xi_{k}^{M}\right\rangle= & \frac{1}{\sqrt{\mathcal{N}\left(\xi_{k}^{M}\right)}}\left|\mathcal{P}_{M}\left(a_{0}, a_{1}, \cdots, a_{M-1}\right)\right\rangle_{P A} \\
= & \frac{1}{\sqrt{\mathcal{N}\left(\xi_{k}^{M}\right)}}\left|a_{j}=i\right\rangle_{P} \otimes \mid \mathcal{P}_{M-1}\left(a_{0}, \ldots,\right. \\
& \left.\left.a_{j-1}, a_{j+1}, \ldots, a_{M-1}\right)\right\rangle_{A}+\frac{1}{\sqrt{\mathcal{N}\left(\xi_{k}^{M}\right)}} \sum_{a_{j} \neq i}\left|a_{j}\right\rangle
\end{aligned}
$$

$$
\otimes\left|\mathcal{P}_{M-1}\left(a_{0}, \ldots, a_{j-1}, a_{j+1}, \ldots, a_{M-1}\right)\right\rangle_{A}
$$

Then the scalar product is

$$
\begin{aligned}
\sum_{k=0}^{n_{M}-1}{ }_{P}\left\langle i \mid \xi_{k}^{M}\right\rangle_{P A} \otimes\left|\xi_{k}^{M}\right\rangle_{C} & \sum_{k^{\prime}=0}^{n_{M-1}-1} \sqrt{\frac{\mathcal{N}\left(\xi_{k^{\prime}}^{M-1}\right)}{\mathcal{N}\left(\xi_{k}^{M}\right)}}\left|\xi_{k^{\prime}}^{M-1}\right\rangle_{P A} \otimes\left|\xi_{k}^{M}\right\rangle_{C} \\
\quad= & \sum_{k^{\prime}=0}^{n_{M-1}-1} \sqrt{\frac{\mathcal{N}\left(\xi_{k^{\prime}}^{M-1}\right)}{\mathcal{N}\left(\xi_{g\left(i, k^{\prime}\right)}^{M}\right)}}\left|\xi_{k^{\prime}}^{M-1}\right\rangle_{P A} \otimes\left|\xi_{g\left(i, k^{\prime}\right)}^{M}\right\rangle_{C}
\end{aligned}
$$

since $k=g\left(i, k^{\prime}\right)$.
[1] C.H. Bennett, G. Brassard, C. Crépeau, R. Jozsa, A. Peres, and W.K. Wootters, Phys. Rev. Lett. 70, 1895 (1993).

[2] D. Bouwmeester, J.-W. Pan, M. Mattle, M. Eible, H. Weinfurther, and A. Zeilinger, Nature (London) 390, 575 (1997); D. Boshi, S. Branca, F. De Martini, L. Hardy, and S. Popescu, Phys. Rev. Lett. 80, 1121 (1998); A. Furusawa, J.L. Sorensen, S.L Braunstein, C.A. Fuchs, H.J. Kimple, and E.S. Polzik, Science 282, 706 (1998).

[3] S. Bose, V. Vedral, and P.L Knight, Phys. Rev. A 57, 822 (1998).

[4] W.K. Wootters and W.H. Zurek, Nature (London) 299, 802 (1982).

[5] M. Murao, D. Jonathan, M.B. Plenio, and V. Vedral, Phys. Rev. A 59, 156 (1999).

[6] W. Dür and J. I. Cirac, J. Mod. Opt. 47, 247 (2000).

[7] S. Popescu (private communication).

[8] V. Bužek and M. Hillery, Phys. Rev. A 54, 1844 (1996); M. Hillery and V. Bužek, ibid. 56, 1212 (1997); V. Bužek, S.L. Braunstein, M. Hillery, and D. Bruß, ibid. 56, 3446 (1997).

[9] V. Bužek and M. Hillery, Phys. Rev. Lett. 81, 5003 (1998).

[10] N. Gisin and S. Massar, Phys. Rev. Lett. 79, 2153 (1997).

[11] D. Bruß, D.P. DiVincenzo, A. Ekert, C.A. Fuchs, C. Macchia- vello, and J.A. Smolin, Phys. Rev. A 57, 2368 (1998).

[12] R.F. Werner, Phys. Rev. A 58, 1827 (1998).

[13] A. Peres, Phys. Rev. Lett. 77, 1413 (1996); M. Horodecki, P. Horodecki, and R. Horodecki, Phys. Lett. A 223, 1 (1996).

[14] N.J. Cerf, Acta Phys. Slov. 48, 115 (1998).

[15] V. Bužek, M. Hillery, and R. Bednik, Acta Phys. Slov. 48, 177 (1998).

[16] S. Massar and S. Popescu, Phys. Rev. Lett. 74, 1259 ().

[17] P.W. Shor, Phys. Rev. A 52, R2493 (1995); A.R. Calderbank and P.W. Shor, ibid. 54, 1098 (1996); A.M. Steane, Phys. Rev. Lett. 77, 793 (1996); R. Laflamme, C. Miquel, J.P. Paz, and W.H. Zurek, ibid. 77, 198 (1996).

[18] C.H. Bennett, B.P. DiVincenzo, J.A. Smolin, and W.K. Wootters, Phys. Rev. A 54, 3824 (1996); E. Knill and R. Laflamme, ibid. 55, 900 (1997).

[19] M. Hillery, V. Buzek, and A. Berthiaume, Phys. Rev. A 59, 1829 (1999); A. Karlsson, M. Koashi, and N. Imoto, ibid. 59, 162 (1999).

[20] R. Cleve, D. Gottesman, and H.-K. Lo, Phys. Rev. Lett. 83, 648 (1999).

[21] A. Barenco, T.A. Brun, R. Schack, and T.P. Spiller, Phys. Rev. A 56, 1177 (1997). 